\title{
THROUGHPUT AND DELAY ANALYSIS OF NEXT-HOP FORWARDING METHOD FOR NON-LINEAR VEHICULAR AD HOC NETWORKS
}

\author{
Ram Shringar Raw ${ }^{1}$ and D. K. Lobiyal ${ }^{2}$ \\ ${ }^{1}$ Ambedkar Institute of Advanced Communication Technologies \& Research \\ rsrao08@yahoo.in \\ ${ }^{2}$ School of Computer and Systems Sciences, Jawaharlal Nehru University \\ New Delhi, India \\ lobiyal@gmail.com
}

\begin{abstract}
Position based routing plays a significant role in multi-hop Vehicular Ad hoc Networks (VANETs), due to high mobility of nodes. Selection of next-hop node is crucial to improve the performance of routing. In this paper, we have proposed a method for selecting next-hop forwarding node based on the distance between the source and next-hop node and link quality. Next-hop node is selected based on Expected Progress Distance (EPD) criteria. The EPD is estimated in terms of expected distance between the source and nexthop node. The expected delay $\left(E_{D}\right)$ and throughput $\left(T_{h}\right)$ are also estimated for the proposed method. The mathematical model derived for calculating EPD, delay, and throughput are simulated in MATLAB and evaluated the performance of the proposed method.
\end{abstract}

\section{KEYWORDS}

VANET, MANET, Next-Hop Forwarding, Position Based Routing, Greedy Forwarding, EPD, Delay, Throughput.

\section{INTRODUCTION}

In 1999, the Federal Communication Commission (FCC) allocated a frequency spectrum for vehicle to vehicle and vehicle to roadside wireless communication (see Figure 1). The Commission then established the Dedicated Short Range Communications (DSRC) service in 2003. DSRC is a communication service that uses the $5.850-5.925 \mathrm{GHz}$ frequency band $(5.9 \mathrm{GHz}$ band) for the use of public safety and private applications [1]. Mobile Ad-hoc networks (MANETs) refer to self-organizing wireless networks consisting of mobile nodes capable to establish communication among them without any fixed infrastructure. Every node in this network acts as router and forwards the message hop by hop. Due to its nature i.e. infrastructurefree environments MANETs can be deployed in emergency rescue, military, airports, sports

DOI : 10.5121/ijans.2012.2204 
stadiums, campus, and disaster management. Due to this broad applications area of MANETs researchers paying more attentions in the development of such networks.

Current advances in wireless communication systems are enabling a new vehicular communication system to improve and protect road transportation. Vehicular Ad Hoc Network (VANET) [2] is the budding and challenging subclass of Mobile Ad Hoc Networks (MANETs) [3]. VANET is composed of a large number of vehicles providing connectivity to each other. We assume that vehicles move in any direction with high mobility. Neighboring vehicles that are within a transmission range directly communicate over a wireless links. End-to-end connectivity between source and destination vehicle in VANET requires multi-hop packet forwarding by intermediate vehicles. VANET supports vehicles that are equipped with computing device, short range wireless interface and GPS receiver [4].

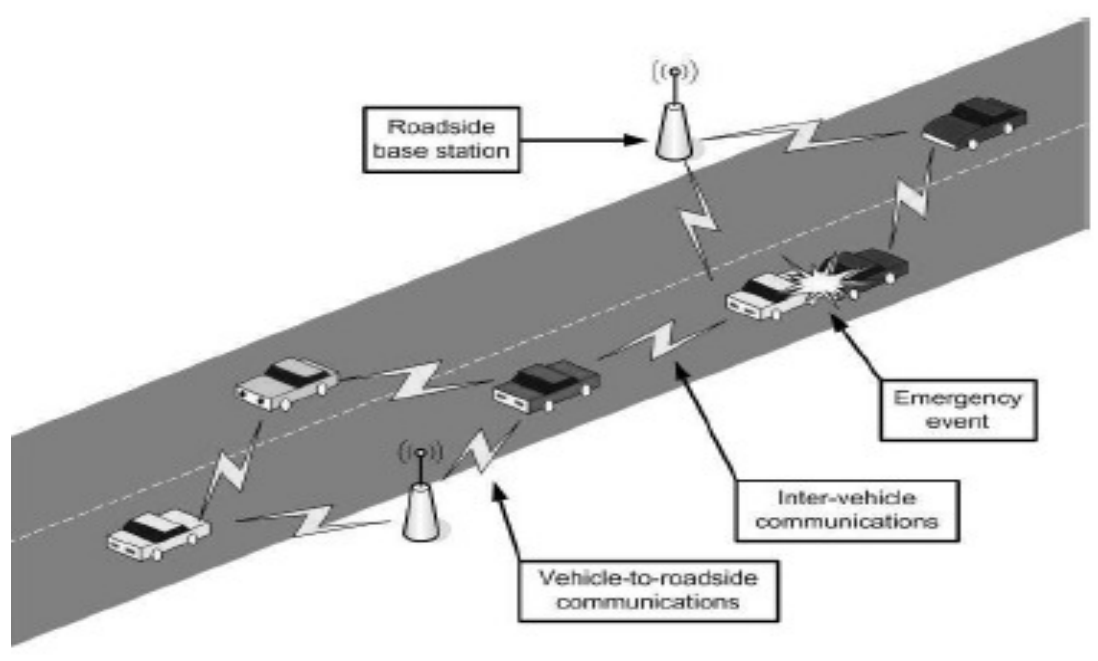

Figure 1. VANET communications

Position based routing can be defined as a next-hop forwarding method in which a node uses the position information of both itself and the destination node to determine a route. Therefore, position based routing protocols does not require any information about the global topology of the network. Unlike traditional routing protocols, position based routing protocols can address the challenges present by interesting properties of VANETs [5].

The dynamic topology of VANET reduces the throughput and efficiency of the routing protocols. Therefore, various approaches have been recommended to improve the throughput and the efficiency of position based next-hop forwarding [6], [7], [8] for linear and non-linear topology network. In this paper, we propose a forwarding method for non-linear network to select the nexthop node using next-hop forwarding method. We also use a link metric that is Expected Progress Distance (EPD) to evaluate the performance of forwarding method.

The rest of this paper is organized as follows. We discuss the related work in section 2. In section 3 , the design of proposed method is introduced. Section 4 presents the mathematical analysis of the proposed method. Results and performance evaluation is presented in section 5. Finally, we conclude this paper in section 6 . 


\section{RELATED WORK}

Takagi and Kleinrock [9] proposed the first position based routing protocol that is MFR routing protocol which is based on the notion of progress. MFR is a well-known loop-free method for finding a route in a network by utilizing position information of nodes [10]. The neighbor with the greatest progress on the straight line joining the source and destination is chosen as next-hop node for sending packets further. Therefore MFR forwards the packet to the node that is closest to the destination node in an attempt to minimize the number of hops [11].

Kranakis [12] proposed the DIR (referred as the Compass Routing) is based on the greedy forwarding method in which the source node uses the position information of the destination node to calculate its direction. Then the message is forwarded to the nearest neighbor having direction closest to the line drawn between source and destination. Therefore, a message is forwarded to the neighboring node minimizing the angle between itself, the previous node, and the destination. A GEDIR [10] is a loop free location based routing algorithm. It is the variant of greedy routing. In GEDIR, a source node forwards packets to its neighbor nodes that are closest to the destination node.

Hiraku, Akira, and Kenichi [13] proposed the next-hop forwarding method by limiting the forwarding distance for linear vehicular ad hoc networks. This is position based routing method based on the greedy forwarding method. In some cases, performance (packet delivery ratio and throughput) of the network may degrade due to longer forwarding distance because longer forwarding distance can cause transmission errors in the wireless network. When the maximum forwarding distance increases, the propagation loss increases and the transmission quality of the wireless link, such as packet delivery ratio degrades. To stop degradation in the network, nexthop forwarding by limiting the forwarding distance method is useful. In this method the author calculated the Expected Progress Distance (EPD) to select the next-hop node by choosing among neighbor nodes that are within a predefined maximum forwarding distance (see Figure 2). This method improves both the forwarding distance and the transmission quality of the wireless link.

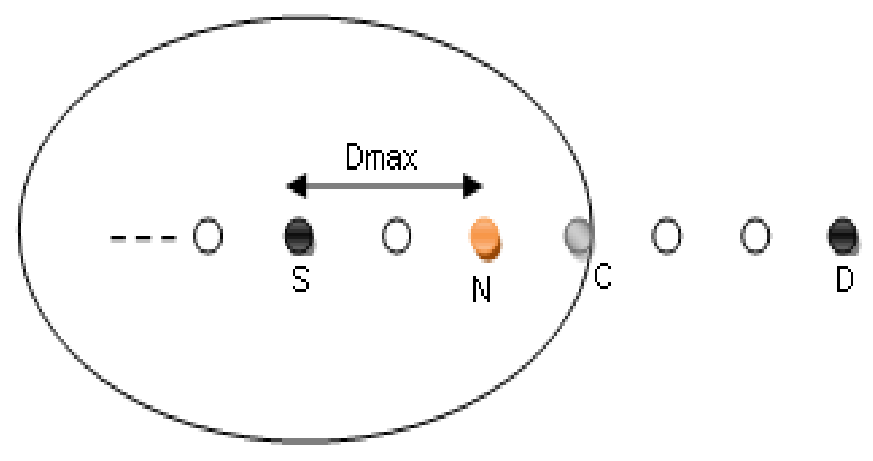

Figure 2. Greedy forwarding by limiting the forwarding distance method (linear case)

\section{PROPOSED WORK}

In this section, we propose a next-hop forwarding method by limiting the forwarding distance for non-linear network. In a dense non-linear network such as city traffic network, nodes are distributed randomly and move in any direction. In such traffic network, numbers of moving and stopped vehicles are very large, spacing between vehicles is small, and fixed road side 
infrastructure is available. However position-based routing in city scenarios faces a lot of challenges due to potentially more irregular distribution of vehicle nodes, forced mobility, and difficult signal reception. City vehicular ad-hoc network defined as dense and large network where source nodes have many alternative paths to destination nodes. The shortest-path positionbased routing protocols frequently select paths that have the minimum number of hops rather than the maximum expected capacity.

\subsection{Next-Hop Forwarding Method by Limiting the Forwarding Distance for Non- Linear Network}

This method considers both forwarding distance and transmission quality to selects a next-hop node. In this method, forwarding node selects a neighbor node as the next- hop node by choosing among neighboring nodes that are within a predefined maximum forwarding distance. The nexthop node is selected using the EPD link metric. This method gives better performance in terms of transmission quality considering Expected Transmission Time (ETT).

In Figure 3, $S$ and $D$ are source and destination nodes. $N$ is the next-hop node and $C$ is the closest to destination node. $D_{\max }$ is the maximum forwarding distance between forwarding node (source node) and next-hop node. As greedy forwarding method decides the next-hop node by exchanging Hello packets and selects the neighbor node that is closest to the destination node as the next-hop node. In this method, a neighbor node with good reception environment and the closest to the destination is selected as next-hop node. As shown in the Figure 3, source node $S$ selects node $N$ (not node $C$ ) as the next-hop node for further transmission.

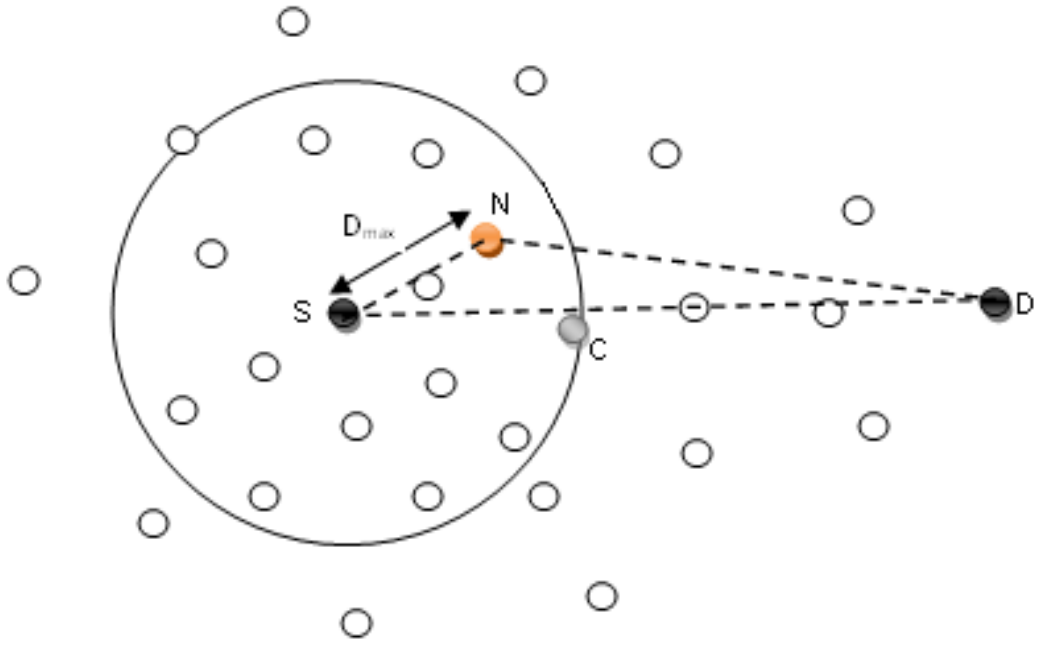

Figure 3. Greedy forwarding by limiting the forwarding distance method (non-linear case)

\subsection{Next-Hop Node Selection}

The progress distance is obtained through position information of forwarding node, neighbor nodes, and destination node. Using the neighbor information table, forwarding node find the nexthop node whose EPD is maximum among all neighboring nodes. As shown in the Fig.2, neighbor node $N$ is selected as the next-hop node. In the greedy forwarding method, neighbor node $C$ is selected as the next-hop node because it is the closest neighbor to the destination node within the 
transmission range. When we consider ETT, the node nearest to the forwarding node (source node) is selected as the next-hop node because smallest ETT gives the best transmission quality of the wireless link in the ad hoc networks.

\section{MATHEMATICAL ANALYSIS}

In this work, we consider the non-linear network where vehicles move on the road in every direction. The distance between nodes is assumed to be a random variable with Poisson distribution. The path loss depends on the forwarding distance between forwarding node and next-hop node. To select next-hop node with good transmission quality, limiting the maximum forwarding distance is more effective. In this section, we study the expected distance to next-hop node $E\left(D_{\max }\right), E T T$, and $E P D$ to select a next-hop node with good transmission quality.

\subsection{Expected Distance to Next-Hop Node}

One of the metrics used in any vehicular network is end-to-to-end delay that depends on the criteria used to select the next-hop node for forwarding the packets. Selecting next-hop node at the maximum distance from the source or close to the destination is to reduce the number of hops occurring in a route. Here it is assumed that the minimum number of hops in a route results in minimum end-to-end delay. Since we have considered a non-linear network of randomly distributed nodes, it is difficult to determine the exact value of maximum distance between a source and next-hop node. Therefore, this distance can be considered as a random variable and its expected value can be determined. Let assumed that a source node $S$ has $n$ neighbors in the direction of destination node. Let $N$ is the next-hop node of source node $S$ (see Fig. 2). Let $\mathrm{d}_{l}, d_{2}$, $d_{3} \ldots, d_{n}$ denotes the distances between source node $S$ and its neighbors [14]. $D_{\max }$ is the maximum distance between the source node $S$ and its one-hop nodes, i.e.

$$
D_{\max }=\operatorname{Max}_{i=1}^{n} d_{i}
$$

We can calculate the expected value of distance $D_{\max }$ between the source node and its neighbors as follows:

Let $F\left(D_{\max }\right)$ and $f\left(D_{\max }\right)$ be the $C D F$ and $P D F$ of $D_{\max }$. Then,

$$
\begin{gathered}
F\left(D_{\max }\right)=P\left[d_{1} \leq D_{\max }, d_{2} \leq D_{\max }, \ldots, d_{n} \leq D_{\max }\right] \\
=\prod_{i=1}^{n} P\left[d_{i} \leq D_{\operatorname{mnx}}\right]=\left(\frac{D_{\max }}{R}\right)^{n}
\end{gathered}
$$

Similarly,

$$
f\left(D_{\max }\right)=\frac{d}{d_{d_{\max }}} F\left(D_{\max }\right)=\frac{n}{R}\left(\frac{D_{\max }}{R}\right)^{n-1}
$$

The expected value of $D_{\max }$ is,

$$
E\left(D_{\max }\right)=\int_{0}^{R} D_{\max } \cdot f\left(D_{\max }\right) d D_{\max }
$$




$$
\begin{aligned}
& =\int_{0}^{R} D_{\max } \cdot \frac{n}{R}\left(\frac{D_{\max }}{R}\right)^{n-1} d D_{\max } \\
= & \frac{n}{R^{n}}\left[\frac{D_{\max }{ }^{n+1}}{n+1}\right]_{0}^{R}=\frac{n A}{n+1}
\end{aligned}
$$

\subsection{Expected Progress Distance (EPD)}

The expected maximum distance between a source and its next-hop node computed in (4) can be used in determining the shortest path between an original source and final destination. Number of hops (hop counts) can be calculated to divide the distance between original source and final destination by the maximum distance obtained by (4). Hop counts is one of the most common routing metric used in most MANET protocols such as AODV, DSR, DSDV, GSR, OLSR and GPSR. But, this is a simple metric to find shortest route between a source and destination.

Hop counts are one equal unit and independent from the quality or other characteristics of the links. Therefore, hop counts though, minimizes the number of hops in the multi-hop network, but it may not minimize end-to-end delay. To take quality of link into account we have also considered transmission time along with the maximum distance between source and next-hop node. Transmission time varies from link to link and thus its exact value cannot be determined. Therefore, we have considered it as a random variable and determined its expected value.

The ETT (Expected Transmission Time) routing metric [15] is an isotonic metric and it is used to maximize the throughput of the path by measuring the link capacities and would increase the overall performance of the network. ETT may be defined in two ways. Firstly, it is defined in terms of Expected number of Transmission (ETX) as follows:

$$
E T T=E T X \cdot \frac{5}{2}
$$

Where $S$ is the size of a packet and $L$ is the bandwidth of the link. ETX [16] is a path metric that is used to maximize the network throughput by measuring loop-free paths and link quality in the wireless network. It is simply the sum of the ETX value of each link along the path. When the ETX metric is lesser for a link, the wireless link is better. In position based greedy forwarding method, ETX is measured by using periodically broadcast control messages which are sent very frequently. Let $p$ is the probability to deliver a packet successfully and $q=1-p$ is the probability of failure to deliver a packet. ETX to successfully deliver a packet to the next-hop [17] can be estimated as:

$$
\begin{aligned}
& E T X=\sum_{n=1}^{\infty} x \cdot p^{x}\left(1-p^{x-1}\right. \\
& =\frac{1}{(1-p)}
\end{aligned}
$$

The relationship between the ETT of a link and ETX can be defined as: 


$$
E T T=E T X: \frac{s}{2}=\frac{S}{L \cdot(1-p)}
$$

To improve the performance network expected distance $E\left(D_{\max }\right)$ calculated in (4) is combined with $E T T$ to compute a new EPD metric for a link. This new metric is used to select a next-hop node to transmit a packet from source to destination. Therefore, link metric, $E P D$ is calculated as expected distance to next-hop node $E\left(D_{\max }\right)$ divided by the expected one-hop transmission time ETT [13] as follows:

$$
\begin{gathered}
E P D=\frac{\text { Expected Distunce to Next Hop Node }}{\text { Expected one hop Transmission Time }}=\frac{E\left(D_{\max }\right)}{E T T} \\
=\frac{n R}{E T T \cdot(n+1)}
\end{gathered}
$$

\subsection{Expected Delay and Throughput}

It is common in VANETs that the nodes move within a certain transmission range. Therefore, network performance directly depends on the number of hops with average delay in a VANET. Delay is one of the key parameter to be considered for vehicular network traffic [18]. It is defined as the time taken for a packet to be transmitted across a network from source to destination. Throughput of the network is universally proportional to the average delay between source and destination [19]. Throughput of the network can be estimated as follows:

$$
\begin{gathered}
\text { Delay }\left(E_{D}\right)=\frac{\text { Expected Progress Distane }(E P D)}{\text { Eandwidth of the Link }(L)}=\frac{n R}{E T T \cdot(n+1)}=\frac{1}{L} \\
\text { Thrughput }\left(T_{R}\right)-\frac{E T T \cdot(n+1) \cdot L}{n R}
\end{gathered}
$$

\section{RESULTS AND PERFORMANCE ANALYSIS}

The mathematical model proposed here has been simulated using MATLAB. In the simulation, we have deployed the nodes randomly. The transmission range of nodes has been fixed at $250 \mathrm{~m}$. The network area is a squared region of $2000 \mathrm{~m}$. This model has been used to calculate the EPD. We have evaluated the impact of network size (i.e., number of nodes in the network) and ETT on Expected Progress Distance. In the simulations, results have been computed in terms of expected progress distance between source and next-hop node.

\subsection{Expected Progress Distance (EPD)}

Figure 4 shows the corresponding result for expected progress distance. From the Figure 4 (a) we can observe that as the number of nodes increases, the expected progress distance between forwarding node and next-hop node is also increases. We have computed the results of EPD for varying network size for different transmission range since it impacts the EPD. The results have been computed by fixing transmission range at different values of $R$, i.e. $250 \mathrm{~m}, 265 \mathrm{~m}$ and $280 \mathrm{~m}$ respectively. As shown in the figure, EPD is better for $R=280 \mathrm{~m}$ than $R=265 \mathrm{~m}$ since higher transmission range provides better value of $E P D$. Further, $E P D$ increases as the node density increases because nodes fall closer and link quality is better between closer nodes. 
International Journal on AdHoc Networking Systems (IJANS) Vol. 2, No. 2, April 2012

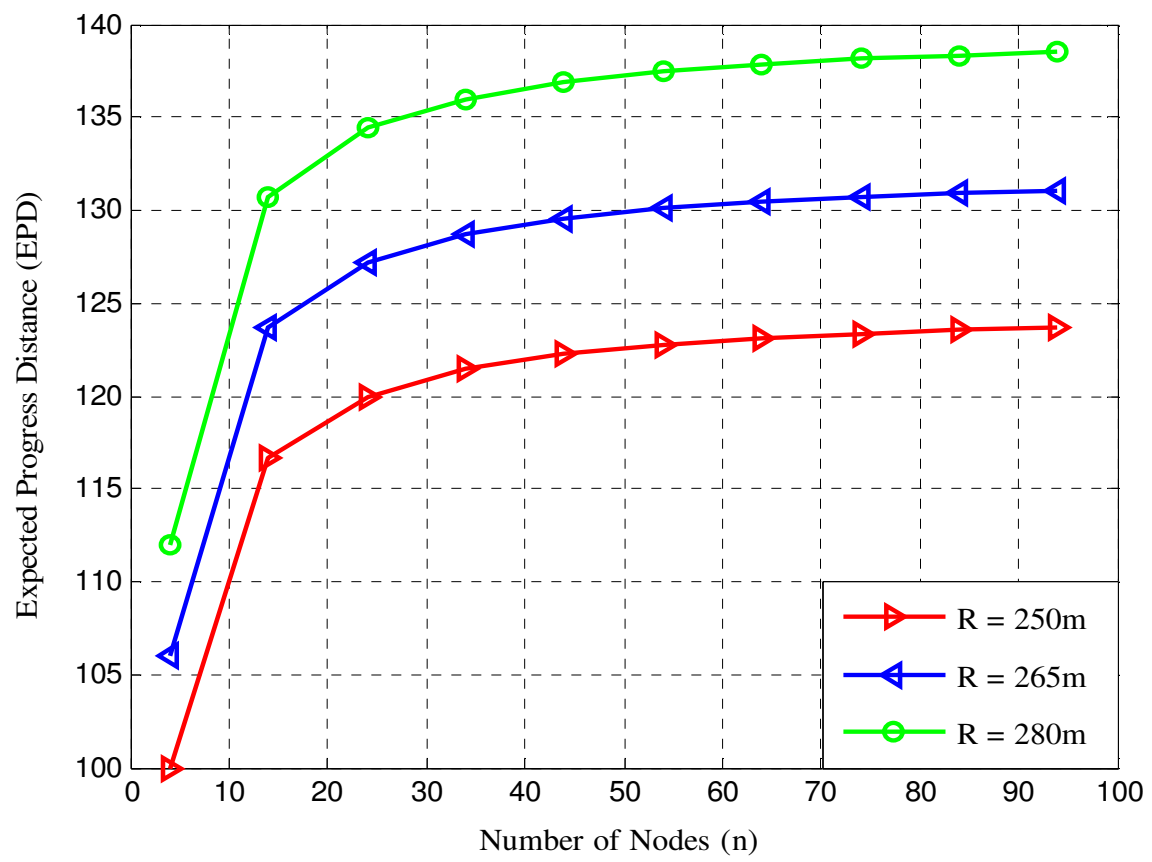

(a)

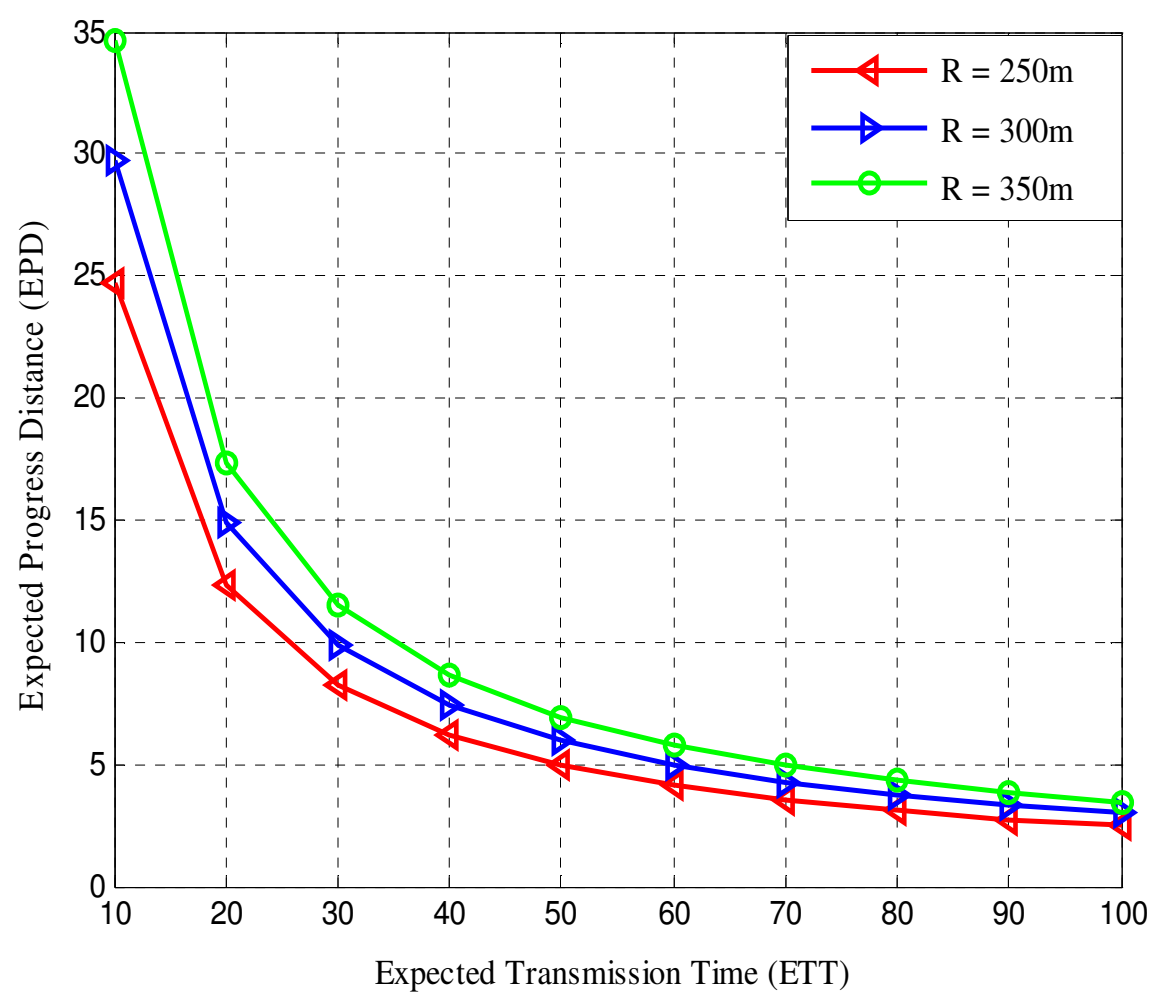

(b)

Figure 4. Expected progress distance $(E P D)$ 
Figure 4 (b) shows the EPD of our proposed method for varying ETT. The results show a significant decrease in the $E P D$ as the $E T T$ increases for a fixed transmission range. The results also shows that as $E P D$ decreases as ETT increases, but $E P D$ is always higher for higher network size $(n=60)$ compared to the lower network size $(n=20)$. This is due to the higher node density that results in reduced value of ETT.

\subsection{Average Delay $\left(\mathbf{E}_{\mathbf{D}}\right)$}

Delay and throughput are most important parameter to be considered for vehicular traffic. Figure 5 (a) shows the variation in average delay as the number of nodes increases. We can observe that as the number of nodes increases, the average delay between forwarding node and next-hop node is decreases. Delay is always higher for higher ETT $(E T T=14)$ compared to lower ETT $(E T T=$ $10)$.

Figure 5 (b) shows the variation in average delay as the packet transmission rate (bandwidth of the link) increases. The results shows a significant decrease in the average delay as the packet transmission rate increases.

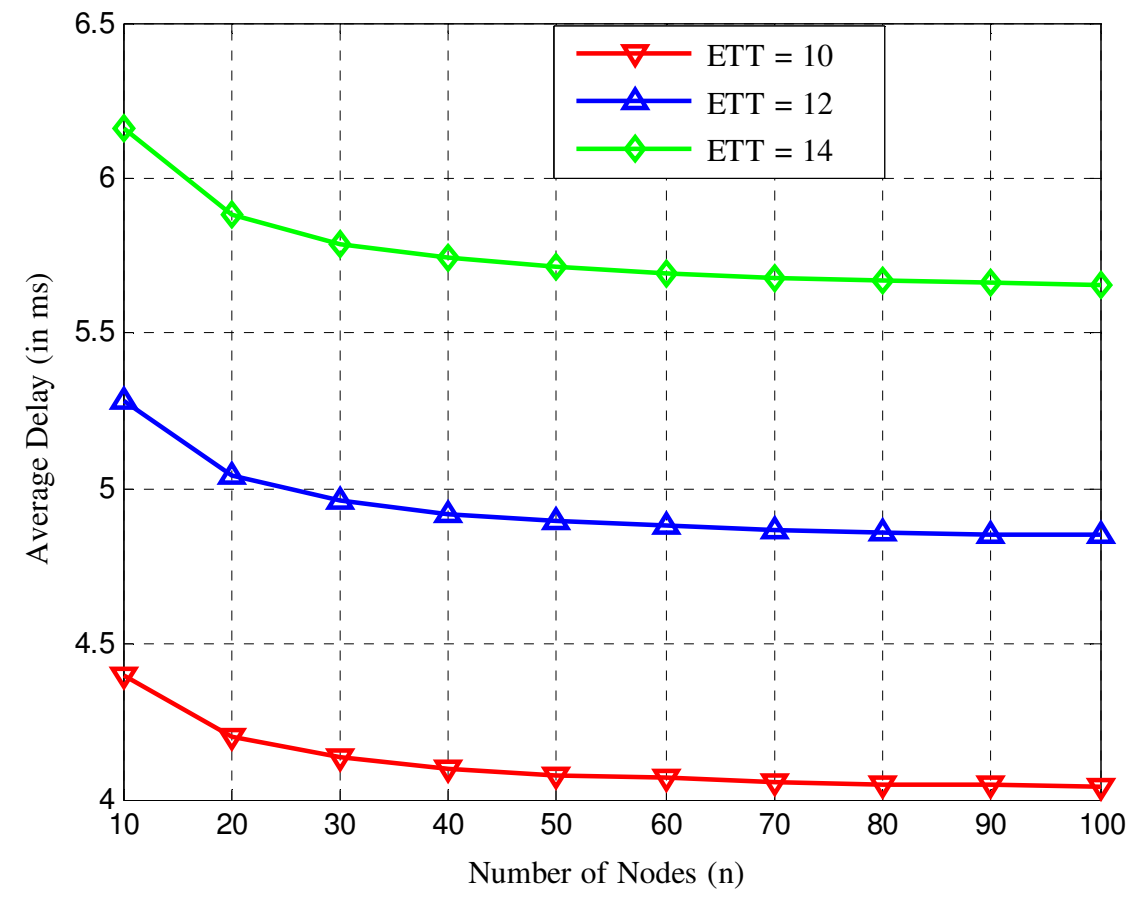

(a) 


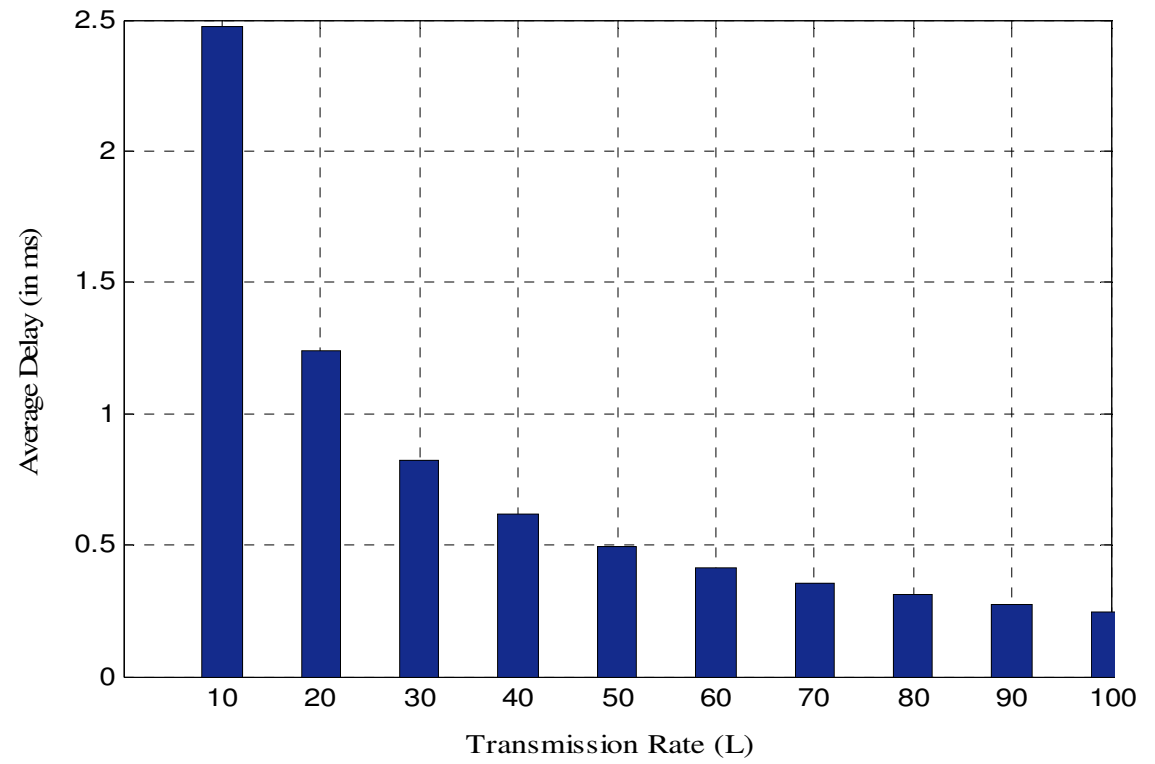

(b)

Figure 5. Average delay as a function of $n$ and $\mathrm{L}$

\subsection{Throughput $\left(\mathrm{T}_{h}\right)$}

Figure 6 shows that number of nodes directly affects the throughput of VANETs communications. From the numerical analysis, we can see that initially throughput increases faster as number of nodes increases and network becomes saturated at 85 nodes and get maximum throughput for the maximum number of nodes for a fixed transmission range.

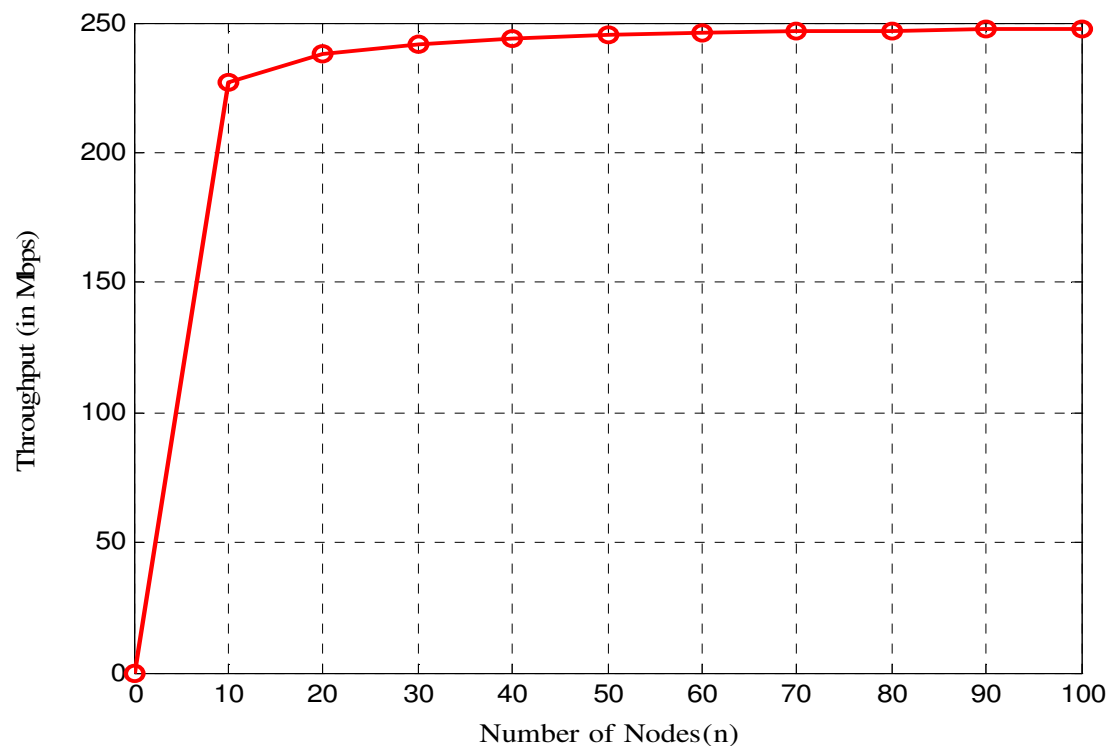

Figure 6. Throughput $\left(\mathrm{T}_{\mathrm{h}}\right.$, in Mbps) 


\section{CONCLUSION}

In this paper, we have proposed a method for selecting next-hop forwarding node based on the distance between the source and next-hop node and link quality. In this method, the neighbor node that has the maximum $E P D$ is selected as the next-hop node among neighboring nodes. Nodes in the network have higher EPD when the network size is large and small ETT. Delay and throughput are estimated for selected next-hop node. Network performance in terms of throughput will be maximized for the maximum number of nodes for a fixed transmission range. Therefore, this method by limiting the forwarding distance is an effective position-based routing method to forward packets from source to destination with good transmission quality of wireless link. Future work will also include the analysis of some other performance metrics for position based routing protocols as they are more suitable in vehicular traffic environment.

\section{REFERENCES}

[1] The FCC DSRC web site. http://wireless.fcc.gov/services/its/dsrc/.

[2] Moustafa, \& Zhang, (2009) Vehicular networks: Techniques, Standards, and Applications. CRC Press.

[3] Murthy \& Manoj, (2008) Ad Hoc Wireless Networks: Architectures and Protocols. PEARSON,ISBN 81-317-0688-5.

[4] S. Manvi, \& M. Kakkasageri, (2008) "Issue in Mobile Ad hoc Networks for Vehicular Communication" IETE Technical Review, Vol. 25, No. 2.

[5] R. S. Raw, S. Das, \& A. Agarwal, (2011) "Thoughts on Vehicular Ad Hoc Networks (VANETs) in the Real World Traffic Scenarios" International Journal Computer Science Management System (IJCSMS) 3(1), pp. 19-26.

[6] R. S. Raw \& D. K. Lobiyal, (2010) "VANET: Position-Based Routing in Urban Environment - A Technical Review" TRANSTEC-2010, New Delhi.

[7] Takano, Okada, \& Mase, (2007) "Performance Comparison of a Position-Based Routing Protocol for VANET" IEEE.

[8] M. Mauve, \& J. Widmer, (2001) "A Survey on Position-Based Routing in Mobile Ad Hoc Networks" IEEE Network.

[9] H. Takagi \& L. Kleinrock, (1984) "Optimal transmission ranges for randomly distributed packet radio terminals" IEEE Transactions on Communications 32 (3), pp. 246-257.

[10] Stojmenovic, Ruhil, \& Lobiyal, (2006) "Voronoi diagram and convex hull based Geocasting and routing in wireless networks" Wireless Communications and Mobile Computing, John Wiley \& Sons Ltd.

[11] R. S. Raw \& D. K. Lobiyal, (2011) "E-DIR: A Directional Routing Protocol for VANETs in a City Traffic Environment" International Journal of Information and Communication Technology (IJICT), ISSN: 1466-6642, Vol. 3 Issue 2, pp.242-257.

[12] Kranakis, Singh, \& Urrutia, (1999) "Compass routing on geometric networks" In: Proc. 11th Canadian Conference on Computational Geometry, Vancouver.

[13] Okada, Takano, \& Mase, (2009) "A Proposal of Link Metric for Next-Hop Forwarding Methods in Vehicular Ad Hoc Networks" IEEE.

[14] C. Yi, Y. Chuang, H. Yeh, Y. Tseng, \& P. Liu, (2009) "Streetcast: An Urban Broadcast Protocol for Vehicular Ad-Hoc Networks".

[15] J. Guerin, M. Portman, \& A. Pirzada, (2008) "Routing Metrics for Multi-Radio Wireless Mess Networks".

[16] Y. Yang, J. Wang, \& Kravets, (2006) "Designing Routing Metrics for Mesh Networks".

[17] B. K. Addagada, V. Kisara, \& K. Desai, (2009) "A Survey: Routing Metrics for Wireless Mess Networks".

[18] H. M. Asif, T. Sheltami, \& E. Shakshuki, (2008) "Power Consumption Optimization and Delay minimization in MANET" Proceedings of MoMM.

[19] L. Hongfei, Y. Zhongiun, W. Tao, L. Lijun, \& Z. Fu, (2010) "Throughput and Reliability Analysis of Information Broadcasting Protocol in VANETs". 
International Journal on AdHoc Networking Systems (IJANS) Vol. 2, No. 2, April 2012

\section{Authors:}

Ram Shringar Raw received his B. E. (Computer Science and Engineering) from G. B. Pant Engineering College, Pauri-Garhwal, UK, India and M. Tech (Information Technology) from Sam Higginbottom Institute of Agriculture, Technology and Sciences, Allahabad (UP), India in 2000 and 2005, respectively. He has obtained his Ph.D (Computer Science and Technology) from School of Computer and Systems Sciences, Jawaharlal Nehru University, New Delhi, India in 2011. He is currently working as Assistant Professor at Department of Computer Science and Engin eering, Ambedkar

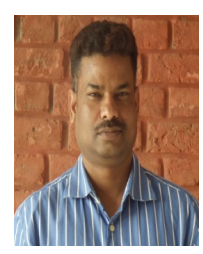
Institute of Advanced Communication Technologies \& Research, GGSIP University, New Delhi, India. His current research interest includes Mobile Ad hoc Networks and Vehicular Ad hoc Networks. Mr. Raw has published papers in International Journals and Conferences including IEEE, Springer, Inderscience, American Institute of Physics, AIRCC, etc.

Daya K. Lobiyal Received his Bachelor of Technology in Computer Science from Lucknow University, India, in 1988 and his Master of Technology and Ph.D both in Computer Science from Jawaharlal Nehru University, New Delhi, India, 1991 and 1996, respectively. Presently, he is an Associate Professor in the School of Computer and Systems Sciences, Jawaharlal Nehru University, India. His areas of research interest are Mobile Ad hoc Networks, Vehicular Ad Hoc Networks, Wireless Sensor Network and Video on Demand.

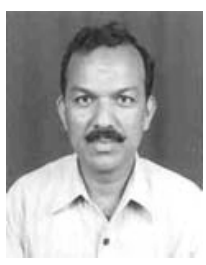

